\title{
Fuzzy-Controlled Living Insect Legged Actuator
}

\author{
Chao Zhang, Feng Cao, Yao Li, Hirotaka Sato* \\ School of Mechanical and Aerospace Engineering, \\ Nanyang Technological University, 639798, Singapore \\ *Corresponding Author. Tel: +65 67905010 \\ E-mail address: hirosato@ntu.edu.sg
}

\begin{abstract}
Steering motor units (e.g. legs) of a living organism by controlled stimulation protocols is a key performance toward living machines, biohybrid robots, or cyborg animals — a fusion of living organisms and man-made devices. To achieve fundamental locomotion pattern generation (e.g. walking gait), a closed-loop (feedback) control system to steer motor units to be set at or to move along a predetermined position and motion path is essential. This study demonstrated the capability to build a precise closed-loop control system manipulating the angular displacement of a coleopteran's leg with electrical stimulation applied directly to the corresponding muscles. We confirmed the correspondence between the angular displacement of the beetle's leg and the electrical stimulation frequency was proportional, nonlinear, and time-variant. A fuzzy control system with multiple membership functions using a proportional controller with adjustable parameters was then proposed and adopted for motion control, and we successfully steered a living leg along a predetermined motion path.
\end{abstract}

Keywords: Fuzzy Control, Biohybrid Robots, Insect Motion Control, Insect Muscle Control 


\section{Introduction}

Inspirations from nature are essential for breakthroughs in robot design. Besides complicated robotic networks with artificial intelligence functions, the emerging production of living machines, biohybrid robots, or cyborg animals [1] targets the combination of merits from both man-made devices and biological organisms, which makes this research field a highly discussed topic of great practical interest. A living machine involves imitation or direct utilization of functional biological elements, from molecules, such as enzymes, cells to tissues, or even to an entire organism, incorporated into artificial context with the desired real-time control [1-3].

What are the merits of using biological organisms for living machines compared with entirely man-made ones? Living organisms, especially insects, possess many excellent properties, including outstanding and unparalleled agility of locomotion, which has made insects among the top focuses in the development of autonomous robots for decades [4-19]. Employing living insects as the platform for legged machines by electrical stimulation on leg muscles to induce user desired motor actions and behaviors leads to significant power-saving. The electrical stimulation of muscle tissues consumes power on the order of a few hundred microwatts, whereas the power consumption of man-made legged robots with mechatronics components is on the order of 100-1000 $\mathrm{mW}$ [7, 20, 21]. Complicated control algorithms, accurate motors, and sensing devices developed with knowledge from bionics are essential for man-made robots to retain their postures and to traverse obstacles. On the contrary, majority of such complicated control algorithms and devices are not necessary for living leggedmachines made of insects' muscle tissues. The user is able to utilize the insect's intrinsic walking system. For example, when the living-legged machine comes across an obstacle, the user shuts off the stimulator and releases the living insect platform from the user's command and control system to let the insect overcome or avoid the obstacle by itself.

Toward living legged 'machine' or 'robot' (walking control), as a starter, we attempt to have legged 'actuator' (one leg motion control). Fine control of one leg motion would finally lead to machine or robot. We have successfully performed control of walking gaits by stimulating insect leg muscles and regulating two legs' motions under predetermined sequence [22]. To regulate leg motion precisely and navigate the machine under control, the motions of each leg and machine itself should be monitored. In laboratory environment, this study used a vision-based feedback where a motion capture system consisting of external cameras is employed to have 3D positional information of the legs. For open environment outside laboratory, it would be possible in future to use MEMS (micro electro mechanical systems) gyroscopes and acceleration meters, which should be attached on insect legs, to obtain feedback information on both angular and linear motions for a closed-loop control. Micro navigation systems for miniature aerial vehicles have been widely deployed [23-28]. Chowdhary et al. integrate off-the-shelf low cost range sensors into a navigation system functional in GPS denied indoor environments [24]. Brown et al. developed a miniature, low cost integrated GPS/inertial system designed to navigate unmanned aerial vehicle in a small self-contained package $<1$ cubic inch including a triad of accelerometers and gyroscopes [23]. Some researchers have attempted to develop tiny electronic nodes which wirelessly communicate with one another or a remote console [29-34] Such a node has self-powering unit (energy scavenger, energy harvester), for which some piezo acoustic power generators, solar cells and biofuel cells has been proposed, designed and demonstrated. Such a selfpowered node can be integrated with MEMS gyroscope, accelerometer and electrical stimulator. Assuming a practically usable self-powered node would be available in future, we would be able to implant or insert such nodes into muscles of interest and make them 
communicate with the remote console at user (probably via a microcomputer mounted on the insect back considering the transmitter of the node should be very low). Such integrated wireless node has not been practically demonstrated yet but the self-powering unit and micro sensors have been designed, manufactured and demonstrated. Once such an integrated wireless node is practically available, we can eliminate wiring electrodes into muscles in insect legged actuators and robots.

The practical use of living machines that directly involves functional biological elements entails three major challenges: (1) using a large biological unit (e.g. living muscle), (2) keeping the biological unit in its functional environmental conditions (e.g. saline medium), and (3) advancing from open-loop control demonstration to the adaptation of a closed-loop (feedback) control system to steer the biological element (e.g. insect leg) to follow a predetermined motion path. As for (1) and (2), a few demonstrations have been conducted for living-muscle-based machines, while various biosensors using small biological units, such as enzymes, antibodies, and living cells, have been developed to date. Akiyama et al. demonstrated the possibility of building motor units by using muscle tissues extracted from living insects and kept in a specially designed chemical solution environment $[2,35,36]$. They demonstrated the chemical stimulation of the insect's muscle tissues. To avoid the maintenance of the working environment, the researchers including the authors did not extract insect muscles but directly used an intact insect leg for building a biological actuator for toward-living-legged machines [20]. Electrical stimulation of large muscle tissues in a living body not only demonstrated the possibility incorporating large biological units of living muscles but also maintained its functional environmental conditions (muscular physiology was self-maintained by the insect as the muscle was not extracted but kept inside the cuticles) for long-term utilization with low power consumption [20, 37]. Furthermore, the researchers also demonstrated a conventional control system with a single proportional controller on muscle motion to steer the leg to follow a user-predetermined motion path [20].

Many researchers have targeted varieties of insect neuromuscular sites for stimulation, including brain, ganglia, nerve cords, sensory units (e.g. antenna, compound eyes), and muscles for achieving locomotion control or appendage motion [4-7, 9-11, 38-44]. Most of the stimulation protocols proposed and tested were conducted in open-loop control manner [4-7, 9-11]. Those researches focused on developing effective stimulation protocols, e.g. to find "What stimulation applied to which neuromuscular sites can elicit desired motor actions and behaviors at how much success rate?". A fundamental issue in the stimulation protocols, where stimulated targets were neural sites other than muscle, was that the stimulation does not guarantee $100 \%$ success rate in inducing desired motion. On the other hand, the muscle stimulation demonstrated in the authors' previous research $[20,22]$ resulted in $100 \%$ success rate in inducing desired leg motions (for example, if the levation muscle is stimulate, no other motion but only the levation motion is induced), in which specific leg muscle groups were individually and separately stimulated via the properly implanted electrodes. Based on such a reliable electrical stimulation targeting leg muscle groups, they demonstrated the abovementioned conventional control system. Despite the successful demonstration, considerably large overshoot, long response time, and severe fluctuation were observed [20]. Hence, for a more sophisticated and precise motion control, it is important to determine the inherent characteristics of an actuator based on biological material (e.g. insect leg muscle), and then to investigate possible solutions.

Functional electrical stimulation (FES), which intentionally induces and regulates desired motor action in limb of polarized patient (or experimental mammals as starter), has been intensively researched. Electrically induced rotation of the limb around the joint can be 
mathematically modelled and controlled by modulating the intensity of stimulation delivered to, for example, the flexor and extensor muscles [45-51]. FES researchers have successfully developed many sophisticated open-loop, rule-based, or feedback controlled FES systems capable of rejecting instantaneous disturbances. Muscle response to electrical stimulation is nonlinear and time-varying, and is also subject to unpredictable reflexes that may generate perturbations [46, 50-52]. Muscle fatigue is also a significant factor in the time-varying nature of the stimulated muscle response [53]. Due to such characteristics, conventional proportional-integral-derivative (PID) controllers adopted for regulating limb rotation have large overshoot, slow rise and settling time, and are sensitive to model mismatch errors [49]. Researchers have then developed fuzzy controls for better performance in regulating limb rotation. Fuzzy control, such as the gain scheduling controller, yielded a slow response with little overshoot $[45,49,50]$. Even though the target animal in this paper is not human nor any other vertebrate but insect, the outcomes from human and vertebrate researches help to develop similar strategies for the insect motion control.

We confirmed a major characteristic of biological material is that, unlike a traditional man-made mechatronics system, the response of biomaterial based on insects (e.g. insect leg muscle) to electrical stimulation can neither be mathematically modeled in practice nor uniquely defined in details [38-44]. There is a clear tendency that the motion response of leg muscles is monotonically increased by the frequency of electrical stimulation with fixed stimulation period to its maximum angular displacement position corresponding to an maximum stimulation frequency ceiling [6, 20, 38-41, 54]. However, an undefined hysteresis property also clearly exists in the leg response, which is demonstrated in details in this paper. Our research in this paper concludes that biomaterial of insect muscle tissues is a nonlinear and time-variant system with hysteresis property. There is a clear difficulty in designing a refined conventional control system based on PID controller for such nonlinear time-variant system in practice. The analytical optimization of the parameters for the conventional PID controller and the stability and robustness of such control system are not guaranteed.

To address the abovementioned problematic characteristic, in this study, we propose to adopt a fuzzy logic for a refined precision feedback control on a biological actuator (e.g. insect's leg motion control). A fuzzy-logic-based control system analyzing analog input values defined in an ambiguous status works better with nonlinear and time-variant systems compared with conventional PID controller [55-58]. Characteristics of the beetle leg muscle as biological materials, such as hysteresis property, make the input values, namely the feedback on angular displacement, defined in an ambiguous relative status in terms of its corresponding stimulation frequency. Clearly, a higher stimulation frequency leads to a larger angular displacement of the beetle's leg motion [20]. However, the standard for a higher stimulation frequency or a larger angular displacement was relatively, rather than absolutely, defined. A fuzzy control system can adjust its internal parameters according to different situations through membership functions.

Another benefit from the fuzzy control system is its simplicity and minimum power requirement for hardware, which is significant as we aim to develop a reliable miniature legged robot with live beetle carrying mechatronic controller on its back. The fuzzy control method does not require convergence of the system. Thus the same design fuzzy control method can be applied on different beetles which do not have exactly the same response to the same electrical stimulation. And the parameters of the fuzzy control method can be quickly calibrated in practice due to its structural simplicity to achieve its optimal performance. 
In this study, we adopted a fuzzy control system for precision motion control of an insect leg. The individual membership function for fuzzification, namely adjustment of internal parameters of a proportional controller, was applied to every single feedback. The fuzzy control system achieved better performance, i.e. smaller overshoot, shorter reaching time (the time between the change in desired input angle and the first reach of the desired angle of the beetle's leg), and less fluctuation, compared with that achieved by a conventional control system using a single proportional controller.

\section{Materials and Methods}

\subsection{Insect for study and electrical stimulation device}

Mecynorrhina torquata (coleopteran order, $6 \mathrm{~cm}$ long and weighing $12 \mathrm{~g}$ on average) was used as the insect platform for the biological actuator. The beetle was placed on a plastic plate and wrapped with dental wax for immobilization. The motion of the beetle's front leg is originally with three degree-of-freedom (DoF), namely, protraction/retraction, levation/depression, and extension/flexion. One DoF motion of protraction/retraction was isolated by inserting two insect pin tips into the corresponding articulations to fix two other joints. The motion of the beetle's front leg was thus reduced to one DoF. One antagonistic pair of muscles on the back of the beetle (Figure 1A) was targeted for electrical stimulation to control the isolated protraction/retraction motion of beetle's front leg with proposed fuzzycontrol system. Four small holes were punched on the pronotum using an insect pin. They were used for two separated channels stimulating the targeted antagonistic pair of muscles. Teflon-insulated thin silver wire was used as the stimulation electrode, with the inserted portion flamed to remove the insulation layer. The length of the inserted portion of the stimulation electrodes was maintained at $2 \mathrm{~mm}$.

The stimulation system consisted of a control computer, a motion-detection system, and a stimulation circuit (Figure 1B). The control computer used our customized software BeetleCommander to control the monophasic square pulse, namely pulse-width-modulation (PWM) wave, as the stimulation signal. The electrical stimulation signal was generated using a custom-programmed microcontroller (Chipcon Texas Instruments CC2431, $6 \times 6 \mathrm{~mm}, 130$ $\mathrm{mg}, 32 \mathrm{MHz}$ clock). The stimulation pulse width was fixed at $1 \mathrm{~ms}$, and the frequency was set between 0 and $200 \mathrm{~Hz}$, because $200 \mathrm{~Hz}$ was already beyond the limitation of the beetle leg levitation [20]. The stimulation voltage was fixed at $1.5 \mathrm{~V}$ using AA batteries. For precision control of a single individual muscle, the stimulating electrical current flow was restricted to only the targeted muscle. However, the biological materials used, i.e. the beetle leg muscles in this case, was internally connected with shared tissue fluid. To achieve that isolation situation, all stimulation channels were isolated using opto-isolators connected with individual AA batteries.

\subsection{Motion-detection system.}

$\mathrm{VICON}^{\circledR}$ vision detection system was adopted for our research to gather feedback information. The motion of beetle's front leg with three makers placed onsite accordingly is monitored by VICON $^{\circledR} 3 \mathrm{D}$ motion-detection system. Two markers placed on the beetle's leg were recognized by the 3D motion-detection system as a solid line segment, and the third marker, placed on the beetle's body, indicated the beetle's position (Figure 1C and 1D). The 3D motion-detection system with 6 cameras is able to detect and store the $\mathrm{X}, \mathrm{Y}$, and $\mathrm{Z}$ coordinates of all markers. The angular displacement was calculated using the formula for angle calculation between two vectors, as follows: 
193

$$
\overrightarrow{\boldsymbol{P}}=\left(\begin{array}{c}
X_{1}-X_{2} \\
Y_{1}-Y_{2} \\
Z_{1}-Z_{2}
\end{array}\right), \quad \overrightarrow{\boldsymbol{P}^{\prime}}=\left(\begin{array}{c}
X_{1}^{\prime}-X_{2}^{\prime} \\
Y_{1}^{\prime}-Y_{2}^{\prime} \\
Z_{1}^{\prime}-Z_{2}^{\prime}
\end{array}\right)
$$

$$
\Delta \theta=\cos ^{-1}\left(\frac{\vec{P} \cdot \overrightarrow{P^{\prime}}}{|\vec{P}|\left|\overrightarrow{P^{\prime}}\right|}\right)
$$

where $\vec{P}$ is the rest neutral position vector, and $\overrightarrow{P^{\prime}}$ is the current position vector, and $\Delta \theta$ is the angle between the rest neutral position and current position vector, namely the angular displacement.

All angular-displacement values were relative and calculated with respect to the leg's initial position (rest neutral position) predefined manually. Before the start of the experiment, the beetle's front leg was placed in position near the middle of its motion range. After that, when the front leg rested and did not move by beetle, we recorded this position as the initial position (rest neutral position), and calibrate the $\mathrm{VICON}^{\circledR} 3 \mathrm{D}$ detection system accordingly. The positive sign of the angular displacement indicated that it was an upswing of the leg's motion relative to the initial position, while the negative sign indicated a downswing motion. Although the leg moves nearly in a 2D plane but technically in a 3D space with an arch trajectory due to stiff but a bit flexible leg structure. The projection of the leg's trajectory on the horizontal plan does not reflect the actual angular displacement but only proportionally reflects the tendency of the angular displacement. The angular displacement calculated in 3D space accurately reflected the movement of the leg. BeetleCommander extracted instantaneous marker position information from the 3D motion-detection system and calculated the immediate leg-motion angular displacement. The 3D motion capturing system consists of six T40s VICON ${ }^{\circledR}$ cameras (four megapixels resolution, 2336 x 1728) operated at 100 frames per second. The accuracy of the motion capturing system was estimated using two markers placed $20 \mathrm{~mm}$ apart on a rigid stick and found $0.51 \pm 0.12 \mathrm{~mm}(2.5 \pm 0.62 \%)$. The rate of marker missing was calculated $0.64 \%$.

\subsection{Fuzzy-Control System for electrically stimulated motion of beetle's leg muscle.}

To achieve the utilization of large biological units in a living organism, which is suggested as a major challenge for practical use of a living machine [1], we proposed the use of a feedback-control system on the PWM wave electrical-stimulation output on the beetle's leg muscles with a fixed 1-ms pulse width and adjustable stimulation frequency. Comparing the properties of biomaterials and conventional man-made devices adopted in biohybrid robots, we suggested that a conventional PID controller is an impractical choice. On the basis of the properties of biomaterials corresponding to electrical stimulation of PWM wave, we accordingly developed a fuzzy control system with multiple membership functions, using the stimulation frequency as a single varying output to control the beetle's leg motion to precisely follow a predetermined motion path. In this paper, we consider the conventional control system with a single proportional controller as a representation. For the fuzzy-control we also proposed to adopt a proportional controller. We demonstrated that the fuzzy controller performs better than the conventional controller. Before investigating these feedback-control systems, in the following section, we characterize, in detail, the properties of the biomaterial (e.g. insect leg muscles) adopted as a large functional element under electrical stimulation. 
Traditional man-made robotic actuators widely adopted in past, which rely on mechatronics systems consisting of electrical, pneumatic or hydraulic motors, have deterministic characters. The relationship between input and output is exact deterministic according to physical laws and mathematical formulas, and it could be locally approximated by linear functions and simplified as a time-invariant system. For example, the torque or the angular acceleration generated by the electrical motor with a fixed input voltage and fixed rotational speed is constant and time-invariant. The linear relationship and the time-invariant system are two key presumptions for a conventional control system.

However, the living biological functional element does not fulfill these presumptions. The inner structure of biomaterials and its chemical reactions as a driving force are yet to be thoroughly studied. The reaction of the beetle leg's angular displacement corresponding to electrical stimulation with a fixed frequency was generalized and empirically studied using experimental data. The experimental result indicated that fuzzy control system is suitable to address this issue.

2.3.1. Graded motion control data. Relationship between the stimulation frequency and the corresponding angular displacement of the beetle's leg was well studied [20]. For the reaction of angular displacement to varying stimulation frequencies in the time domain, the stimulation frequency was fixed and the angular displacement of the beetle's leg was recorded from its initial position to its maximum position with respect to time. For the maximum angular displacement in the frequency domain, only the maximum angular displacement of the beetle's leg under each fixed stimulation frequency was recorded. Statistical analysis of the mean value and deviation was studied.

The stimulation voltage was fixed at $1.5 \mathrm{~V}$ for all experiments $(10-100 \mathrm{~Hz}, 0$ to $0.5 \mathrm{~s}, \mathrm{~N}=$ $5, \mathrm{n}=25$. Unless otherwise stated, $\mathrm{N}$ refers to the number of beetles used for each feedback control experiment and $\mathrm{n}$ refers to the number of trials for overall individual beetles in this paper). The electrical stimulation was applied directly on beetle's leg muscle via silver wire at fixed frequency from $10 \mathrm{~Hz}$ to $100 \mathrm{~Hz}$ with $10 \mathrm{~Hz}$ interval. Five individual beetles were used for each experiment, and each experiment of electrical stimulation at fixed frequency was repeated five times.

The angular displacement reaction of the beetle's leg corresponding to a fixed stimulation frequency $(10-100 \mathrm{~Hz}, 0$ to $0.5 \mathrm{~s}, \mathrm{~N}=5, \mathrm{n}=25)$ was studied in the time domain [20]. The graded motion analysis shows a strong time-dependent relationship between the electrical stimulation frequency and the leg's angular displacement. The concave curve of the angular displacement indicates a decreasing angular velocity with respect to time. In addition, the maximum angular displacement reaction of the beetle's leg corresponding to a fixed stimulation frequency $(20-100 \mathrm{~Hz}, \mathrm{~N}=5, \mathrm{n}=25)$ was studied in the frequency domain. The angular displacement reached its maximum position after $0.5 \mathrm{~s}$. The graded motion data also shows a well-established proportional relationship between the electrical stimulation frequency and the leg's angular displacement. The experiment result showed that the living biological functional element is a nonlinear, time-variant system.

The graded motion analysis [20] indicate that the larger the difference in the stimulation frequency, the larger the difference in the time required for the beetle's leg to reach the maximum angular displacement position. This implies that during continuous stimulation, a larger change in the stimulation frequency results in a longer time required by the beetle's leg to reach the maximum angular displacement position. Moreover, only after the beetle's leg reaches the maximum angular displacement position, we can gather valid feedback information on the beetle leg's angular displacement by using a 3D motion-detection system. 
Therefore, the system requires the membership function to adjust the update time interval on the basis of each desired change in the output value. The update time interval is defined as the total time of the current stimulation wave before the next update of the stimulation frequency. It is mainly designed to be no shorter than the time that the beetle leg takes to reach its final position, under a certain stimulation frequency. It can be deduced from the graded motion control data [20].

2.3.2. Hysteresis motion data. The graded motion result only provided the relationship between the stimulation frequency and the levitation angle starting from the same initial position with the same initial muscle length. This hysteresis test targeted the angular displacement of the beetle's front leg starting from a neutral position under electrical stimulation with the stimulation frequency continuously increasing from 10 to $120 \mathrm{~Hz}$, and the angular displacement from the maximum angular displacement position under electrical stimulation with the stimulation frequency continuously decreasing from 120 to $10 \mathrm{~Hz}$. Before the experiment, the leg was placed and released at rest around middle of the angular displacement range without any electrical stimulation as neutral beginning position which is set at zero degree. The angular displacement of the leg was measured relatively to that neutral position.

The experiments with increasing and decreasing stimulation frequency were continuously performed successively without pause. The stimulation frequency was increased from 10 to $120 \mathrm{~Hz}$, with a $10 \mathrm{~Hz}$ step, and then was decreased from 120 to $10 \mathrm{~Hz}$, with a $10 \mathrm{~Hz}$ step. The stimulation at each frequency was maintained for $800 \mathrm{~ms}$ for the beetle's leg muscle to reach its saturation position. We targeted protraction/retraction muscles, an antagonistic pair of muscles, and stimulated these muscles. For example, when the protraction muscle was stimulated, the retraction muscle was reserved intact (no stimulation) so that the retraction muscle did not exert force on the joint and the induced angular displacement was solely due to the electrical stimulation of the protraction.

The angular displacement with increasing and decreasing stimulation frequency (10-120 $\mathrm{Hz}, \mathrm{N}=5, \mathrm{n}=25$ ) demonstrated that the targeted biomaterial, namely the beetle's leg-muscle tissue, exhibits strong hysteresis property. The detailed data gathered in the experiment was thoroughly studied with statistics in the following results section.

\subsubsection{Theoretical analysis of conventional control system with a single proportional} controller. We adopted a conventional control system with a single proportional controller as a representation of a conventional control system. A conventional control system with a single proportional controller is theoretically equivalent to a fuzzy control system with a single membership function. The experimental results of the single proportional controller system were gathered for further study of general properties of the proportional controller. The effect of the membership function on the fuzzy control system was studied and revealed on the basis of the results, which were used for preparing the fuzzy control system designed with multiple membership functions.

The concept of proportional control was used to adjust the magnitude of the step increment and decrement of the stimulation frequency according to the following function:

$$
f_{\text {out }}{ }^{*}=f_{\text {out }}+K_{p} \times e(t)
$$

where

$f_{\text {out }}$ is the last output stimulation frequency from the proportional controller.

$f_{\text {out }}{ }^{*}$ is the updated output stimulation frequency from the proportional controller. 
$\mathrm{K}_{\mathrm{p}}$ is the proportional gain (adjustable by user).

$\mathrm{e}(\mathrm{t})$ is the instantaneous angular displacement error at time $\mathrm{t}$.

The initial output stimulation frequency was set to $2 \mathrm{~Hz}$, and a maximum ceiling of $120 \mathrm{~Hz}$ was adopted for the output stimulation frequency. The graded motion data and the hysteresis graph demonstrated that the effect of the stimulation frequency below $2 \mathrm{~Hz}$ is basically negligible, and that the leg angular displacement reaches its maximum motion range with the stimulation frequency beyond $120 \mathrm{~Hz}$ (Figure 2). The minimum frequency for levitation stimulation is $2 \mathrm{~Hz}$, which means there is always stimulation for the levitation leg muscle. The minimum frequency for counterpart stimulation is $0 \mathrm{~Hz}$, which means there can be no stimulation for the counterpart leg muscle.

The electrical stimulation frequency from one channel initially increased to elicit the leg to move to the desired position. If the actual leg motion angle became larger than the predefined angle, the stimulation from the current working channel continued to decrease its stimulation frequency, whereas the stimulation from the counter-channel started decreasing the motion angle by increasing the corresponding stimulation frequency. Similarly, if the actual motion angle became smaller than the predefined angle, the counter-channel stopped generating the stimulation signal and the previous working channel started to increase its stimulation frequency to increase the leg motion angle. As such, the two channels that stimulated the antagonistic pair of muscles worked simultaneously to ensure that the beetle's leg followed the predetermined motion path.

The detected angle displacement, the desired angle displacement, and their difference were used as the input parameters. The proportional gain of the controller and the update time interval were used as the output parameters. On the basis of the analysis of the results from the conventional control system with a single proportional controller, the relationship between the input and output parameters was well studied. The membership function of the fuzzy control system changed both the value of proportional gain and the update time interval to account for the angular motion of the leg. The fuzzy control system with multiple membership functions was designed in accordance with our findings on the relationship between these two key factors and the experiment results.

\subsubsection{Theoretical analysis of fuzzy control system with multiple membership functions. A} fuzzy control system conceptually consists of three stages. An input stage, which reads feedback sensor values and then maps sensor or other inputs to the appropriate membership functions; a processing stage, which invokes the predefined membership functions and then calculates the result; and an output stage, which converts the result from the processing stage into a specific control output value. For the output stage, we used a proportional controller for the feedback control system. For the processing stage, we used the membership function to adjust the proportional gain of the controller and other parameters of the control system.

In the input stage, the input parameters include the detected angular displacement, and desired angle displacement. These two parameters are mapped to the appropriate membership functions. The proposed shape of the membership functions is triangular. We selected the same point as the start and end of each detected angle displacement for the triangular membership functions. Consequently, each detected angle displacement had one unique corresponding membership function. As a result, at each detected angle displacement, the membership function gives one unique proportional gain for the output stage.

On the basis of the relationships between the key factors and the feedback control results, the corresponding membership functions could be designed as follows: 
(a) The system adopts a larger proportional gain for a larger angle difference between the detected and desired angle displacements. Proportional gain also increases with a larger desired angle input. Two variables as input, namely the angular difference between the detected and desired angular displacements $\left|\theta_{\text {Desired }}-\theta_{\text {Current }}\right|$, and the desired angular displacement $\theta_{\text {Desired }}$, and one dependent variable, namely $\mathrm{K}_{\mathrm{P}}^{*}$, as output are for the membership function. For simplicity, efficiency, reliability, and general applicability, we adopted a linear relationship of the increase in proportional gain. No other relationship is justified theoretically to have better performance than a simple linear relationship. It has the general linear relation form between the input and output as $Y=\beta_{0}+\beta_{1} X_{1}+\beta_{2} X_{2}$, according to the function below.

$$
K_{p}^{*}=K_{p_{\text {base }}}+a_{K_{p}} \times\left|\theta_{\text {Desired }}-\theta_{\text {Current }}\right|+b_{K_{p}} \times \theta_{\text {Desired }}
$$

$\mathrm{K}_{\mathrm{p}}{ }^{*}$ is the $\mathrm{P}$ controller value for the next frequency update

$\theta_{\text {Desired }}$ is the desired angle of motion used as the input

$\theta_{\text {Current }}$ is the current angle of motion detected by the VICON ${ }^{\circledR} 3 \mathrm{D}$ system

$\mathrm{K}_{\mathrm{p}_{\text {base }}}, \mathrm{a}_{\mathrm{K}_{\mathrm{p}}}$, and $\mathrm{b}_{\mathrm{K}_{\mathrm{p}}}$ are parameters

$\mathrm{K}_{\mathrm{p}_{\text {base }}}+\mathrm{a}_{\mathrm{K}_{\mathrm{p}}} \times\left|\theta_{\text {Desired }}-\theta_{\text {Current }}\right|$ is the linear relationship between proportional gain and angle difference

$\mathrm{b}_{\mathrm{K}_{\mathrm{p}}} \times \theta_{\text {Desired }}$ is the increase in proportional gain from a larger desired angle input

(b) The system adopts a longer update time interval for a larger angle difference between the detected and desired angle displacements. The update time interval also increases with a larger desired angle displacement. For the same reason and in the same form as $K_{P}^{*}$, we adopted a linear relationship for the membership function of $T^{*}$, according to the function below.

$$
T^{*}=T_{\text {base }}+a_{T} \times\left|\theta_{\text {Desired }}-\theta_{\text {Current }}\right|+b_{T} \times \theta_{\text {Desired }}
$$

$\mathrm{T}^{*}$ is the update time interval for the next frequency update

$\theta_{\text {Desired }}$ is the desired angle of motion used as the input

$\theta_{\text {Current }}$ is the current angle of motion detected by the VICON $^{\circledR} 3 \mathrm{D}$ system

$\mathrm{T}_{\text {base }}, \mathrm{a}_{\mathrm{T}}$, and $\mathrm{b}_{\mathrm{T}}$ are parameters

$\mathrm{T}_{\text {base }}+\mathrm{a}_{\mathrm{T}} \times\left|\theta_{\text {Desired }}-\theta_{\text {Current }}\right|$ is the linear relationship between update time interval and angle difference

$\mathrm{b}_{\mathrm{T}} \times \theta_{\text {Desired }}$ is the increase in update time interval from a larger desired angle input

The membership function parameters include proportional gain and update time interval, which are determined by the angle difference and desired angle displacement according to functions (3) and (4), respectively. The processing stage invokes the unique corresponding membership function, and generates the corresponding stimulation frequency and the update time interval according to function (2). Finally, in the output stage, the microcontroller converts the stimulation frequency and update time interval into corresponding PWM waves.

For the hysteresis properties of the beetle's leg muscle under electrical stimulation, we generally consider that the corresponding stimulation frequency of a certain angular displacement is in a fuzzy status. Considering the motion direction of the beetle's leg, we can narrow this fuzzy status into a smaller range of frequencies according to the hysteresis property graph shown in Figure 2. Such additional modules, e.g., adding binary factors of motion direction into equation (3), may improve the fuzzy control system in theory for future development. 


\subsection{Experimental setup}

The first set of experiments was performed using the conventional control system with a single proportional controller to achieve a preset angular displacement of $20^{\circ}$. We adopted a conventional control system with a single proportional controller having proportional gain values ranging from 0.5 to 1.0 , with 0.1 increments.

The second set of experiments was performed using a fuzzy control system with multiple membership functions to achieve a preset angular displacement of $20^{\circ}$. Individual beetles same as those used in the conventional control system were tested for this experiment.

From the result of the first set of experiments, which used conventional control system, we deduced the lower and upper bounds for the value of proportional gain, as well as the update time interval for each value of proportional gain, and hence established a linear relationship between the update $\mathrm{P}$ controller proportional gain value and the update time interval.

Kp values are adjusted according to equation (3), where $K_{P}^{*}$ represents the real-time calculated $\mathrm{Kp}$ value for the proportional controller. There are two types of parameters. The first are user input parameters, namely $\theta_{\text {Desired }}, b_{\mathrm{k}_{\mathrm{p}}}$, the calibration value 1 and 2 for $\mathrm{Kp}$ with corresponding value 1 and 2 for $\left|\theta_{\text {Desired }}-\theta_{\text {Current }}\right|$. The second are values calculated from calibration, namely $\mathrm{K}_{\mathrm{p}_{\text {base }}}, \mathrm{a}_{\mathrm{K}_{\mathrm{p}}}$. Before the experiment, the user inputs the values of the user input parameters based on conclusions from previous experiments. Our previous experiment showed that the proportional gain should be larger than 0.5 and smaller than 1 in order to have the closed-loop control work in practice. When the proportional gain was smaller than 0.5, the beetle's leg did not reach the predetermined position. When the proportional gain was larger than 1, the fluctuation did not settle at the predetermined path. The largest absolute angular displacement range was around 40 degree for both upward and downward movements, which depended on both the experiment setup and individual beetle [20]. We use these values as boundary conditions. The proportional gain $\mathrm{K}_{\mathrm{P}}^{*}=1.0$ is corresponding to the largest angular difference $\left|\theta_{\text {Desired }}-\theta_{\text {Current }}\right|=40$. The proportional gain $\mathrm{K}_{\mathrm{P}}^{*}=0.6$ is corresponding to the angular difference $\left|\theta_{\text {Desired }}-\theta_{\text {Current }}\right|=5$. The parameter $b_{K_{p}}=0.02$ is empirically optimized from our experiment result. These values were empirically optimized values from various experiment to have our presented experiment result.

After that, we use calibration, according to the following conditions 1 and 2.

$\mathrm{K}_{\mathrm{P}}^{*}=1.0$ according to equation (3) when $\left|\theta_{\text {Desired }}-\theta_{\text {Current }}\right|=40 \quad$ (Condition 1 )

$\mathrm{K}_{\mathrm{P}}^{*}=0.6$ according to equation (3) when $\left|\theta_{\text {Desired }}-\theta_{\text {Current }}\right|=5 \quad$ (Condition 2)

to calculate the values for the rest of the parameters $K_{p_{\text {base }}}$ and $\mathrm{a}_{\mathrm{K}_{\mathrm{p}}}$. We have two unknown parameters in equation (3). We have to conditions to calculate the unknown parameters.

Tp values are adjusted according to equation (4) in the same way as Kp in equation (3). The values of four parameters are also obtained in the same way as for the $\mathrm{Kp}$ in equation (3) based on our previous experiment result [20]. Before the experiment, the user inputs the values of the user input parameters based on conclusions from previous experiments. Our empirically optimized result is that the largest update time interval $\mathrm{T}^{*}=400$ is corresponding to the largest angular difference $\left|\theta_{\text {Desired }}-\theta_{\text {Current }}\right|=40$. The update time interval $\mathrm{T}^{*}=100$ is corresponding to the angular difference $\left|\theta_{\text {Desired }}-\theta_{\text {Current }}\right|=5$. The parameter $b_{\mathrm{T}}=1$ is empirically optimized from our experiment result.

After that, we use calibration, according to the following conditions 3 and 4 . 
$\mathrm{T}^{*}=400$ according to equation (3) when $\left|\theta_{\text {Desired }}-\theta_{\text {Current }}\right|=40 \quad$ (Condition 3)

$\mathrm{T}^{*}=100$ according to equation (3) when $\left|\theta_{\text {Desired }}-\theta_{\text {Current }}\right|=5 \quad$ (Condition 4)

to calculate the values for the rest of the parameters $\mathrm{T}_{\mathrm{p}_{\text {base }}}$ and $\mathrm{a}_{\mathrm{T}}$. We have two unknown parameters in equation (4). We have to conditions to calculate the unknown parameters.

During the experiment, we have feedback of the current angular displacement $\theta_{\text {Current }}$. With all the values of the parameters known, we can thus calculate the values of $\mathrm{K}_{\mathrm{p}}^{*}$ and $\mathrm{T}^{*}$ accordingly.

\subsection{Hardware implementation of the fuzzy control system.}

We developed customized software BeetleCommander for the fuzzy feedback control system. Electrical stimulation signals generated from two separate stimulation channels were used to control one antagonistic pair of muscles. BeetleCommander extracted instantaneous markerposition information from the $3 \mathrm{D}$ motion-detection system and calculated the immediate legmotion angle. The protraction/retraction feedback motion control was adopted as an example to explain the working principle of our feedback control system, which was controlled by two separate channels from BeetleCommander. Electrical stimulation signals from the two channels were automatically generated according to the desired motion angle/path input. Both channels of stimulations worked independently and concurrently.

For the fuzzy control system, the input consisted of three parameters: the detected angle displacement from the 3D motion-detection system, the user input desired angle displacement, and the angle difference between the detected and desired angles. The output comprised two parameters: the output frequency for the PWM wave and the update time interval.

The graded motion control results show the minimum time required to reach the maximum angular displacement position with respect to the stimulation frequency. The relationship between the minimum time required, namely the update time interval, and the stimulation frequency is nondeterministically proportional. Together with the hysteresis test results, the data show that the relationship between the angular displacement and the stimulation frequency is also nondeterministically proportional. For ease of implementation, we simplified these nondeterministically proportional relationships into proportional relationships using adjustable parameters.

The output frequency of the fuzzy control system was non-integer because of the noninteger feedback data and non-integer proportional controller gain. For the convenience of electrical stimulation with no measurable difference, we used all integer values for the output stimulation frequency. To achieve that, we replaced the output frequency with its nearest integer number.

\subsection{Data analysis and statistics.}

In the data analysis of the experiments described herein, $\mathrm{N}$ refers to the number of beetles used for each feedback control experiment and $\mathrm{n}$ refers to the number of trials for overall individual beetles. Values are given as mean \pm standard deviation. Overshoot was calculated as absolute angular displacement as well as in percentage form. The mean overshoot, as well as the mean reaching time, was calculated as an average over $n$ number of trials.

\section{Results and Discussion}

3.1. Muscle reaction to electrical stimulation is a nonlinear, time-variant system with hysteresis property. 
The hysteresis motion data in Figure 2 indicate that for the same difference in angular displacement, the difference in the stimulation frequency is large when the absolute angular displacement is very large or very small, approximately above $25^{\circ}$ or below $15^{\circ}$, and that the difference in the stimulation frequency is small when the absolute angular displacement lies in the middle range, from approximately 15 to $25 \mathrm{~Hz}$. Consequently, a fast change in the stimulation frequency is required when the absolute angular displacement is very large or very small, and a slow change in the stimulation frequency is required when the absolute angular displacement is in the middle range. Such a property does not exist in a time-invariant linear system. Thus, to realize this preferred result, the system must adopt membership functions to adjust the controller parameters, mainly the proportional gain for a proportional controller, on the basis of the feedback information.

Results in the frequency domain, shown in Figure 2, indicated that when the beetle's leg moves away from the neutral position, the stimulation frequency increases slowly at the beginning and then rapidly for the same increase in angular displacement, while in case of movement toward the neutral position, the stimulation frequency decreases slowly at the beginning and then rapidly for the same decrease in angular displacement. To reach the same angular displacement, the corresponding frequency of the motion type moving toward the neutral position differs from that of the motion type moving away from the neutral position.

Because a significant difference exists in the stimulation frequencies required to reach the same angular displacement between the movement away from and the neutral positon and that toward it (Figure 2), the detected angular displacement does not clearly indicate the exact corresponding value of the stimulation frequency. For example, $80 \mathrm{~Hz}$ was considered as higher stimulation frequency with larger angular displacement for the movement away from the neutral position, with corresponds to an approximately $25^{\circ}$ angular displacement. On the other hand, $60 \mathrm{~Hz}$ was considered as the higher stimulation frequency with larger angular displacement for the movement toward the neutral position, which corresponds to an approximately $30^{\circ}$ angular displacement.

The deviation in the angular displacement is much smaller for the increasing stimulation frequency as compared with that for the decreasing stimulation frequency. The deviation is accumulative because the experiment is continuously ongoing with variable stimulation frequency. Furthermore, the electrical stimulation effect with varying frequency has minimum and maximum limitations, which depend on the beetle's own properties and are unique for each beetle; yet they are, in general, approximately $20 \mathrm{~Hz}$ and $100 \mathrm{~Hz}$, respectively (Figure 2). The proportional relationship between the stimulation frequency and swinging angular displacement was effectively established only on a partial span of the entire simulation frequency.

We considered the above mentioned properties, and the fact that the internal biological working mechanism and chemical reaction of beetle muscle is difficult to be mathematically modeled. Thus, we reached the conclusion that a simplified linearized relationship between the stimulation frequency and angular displacement, angular velocity, or angular acceleration of the leg motion is not able to be established in practice. Those three parameters of the leg motion are also strongly dependent on reaction time, which was demonstrated in the graded motion data [20] and Figure 2.

These results conclude that the biomaterial of insect muscle tissues is a nonlinear and time-variant system. And the experiment result showed that the living biological functional element exhibits an unclearly defined fuzzy relationship between the electrical stimulation frequency and angular displacement of the beetle's leg. 

predetermined path, but the latter performs better.

On the basis of the relationship between the electrical stimulation frequency and the beetle leg's angular displacement, we used the concept of fuzzy control to steer the leg movement according to a preset angular displacement and hence follow a predetermined motion path, which was separated into a series of consecutive preset angular displacements. Figure 3A and 3B shows the schematic representation of both conventional and fuzzy control systems.

The conventional control system with a single proportional controller was adopted for the experiment $\left( \pm 20^{\circ}\right.$ step function, $\left.t=10 \mathrm{~s}, \mathrm{~N}=5, \mathrm{n}=30\right)$. Figure 4 demonstrates that such a control system can enable the beetle's leg motion to follow the preset angular displacement closely. With an increment in the proportional gain of the controller $(\mathrm{Kp})$ from 0.5 to 1.0 , the leg response overshoot generally increases, whereas the settling time decreases. The fuzzy control system with multiple membership functions was adopted for the experiment $\left( \pm 20^{\circ}\right.$ step function, $\mathrm{t}=10 \mathrm{~s}, \mathrm{~N}=5, \mathrm{n}=30$ ). Figure $5 \mathrm{~A}$ demonstrates that the fuzzy control system can also enable the beetle's leg motion to follow the preset angular displacement closely. It also shows the stimulation frequency in the corresponding time domain.

Three major properties of the two control systems - overshoot displacement, reaching time, and damping effect - were compared. Figure 5B illustrates how the values of overshoot displacement and reaching time were determined. The overshoot displacement is defined as the first peak value of the detected motion path. Because the beetle is alive and can steer its own legs' angular displacement, we selected the first peak value to avoid extreme cases where the first peak value as overshoot is not the maximum value. The reaching time is defined as the time between the change in the desired input angle and the first reach of the desired angle of the beetle's leg. The living functional element may not settle into the predetermined path but rather fluctuate around it because of the internal noise from the beetle's own movement. Thus, with such a possibility, the reaching time is a significant indicator of the system's response rate. The damping effect is defined as the fluctuation in the beetle leg's angular displacement around the predetermined path.

The controlled leg movement exhibits the following three interesting properties. (1) The overshoot angular displacement. (2) The reaching time, instead of settling time, which is the time span between the start of the input angle and the first time the system reaches the input angle; because of the obvious fuzzy system property and disturbance from the beetle itself, the settling time may not accurately reflect the system's response time, or there may not even be a settling time because of the fluctuation. (3) The damping effect, which is the fluctuation in the controlled motion.

On the basis of the above presented experimental results in Figure 5A, we conclude as follows: With increasing proportional gain, the experimental results demonstrate decreasing steady-state error, decreasing reaching time, and increasing damping and increasing overshoot. With increasing update time interval, the experimental results demonstrate decreasing damping and increasing reaching time.

The fuzzy control system with multiple membership functions minimizes these disadvantages and has the closest control fit for the predetermined leg motion path. Figure 6A and $6 \mathrm{~B}$ show a statistical analysis of the comparison of the overshoot and reaching time between a conventional controller with a single proportional controller and a fuzzy control system with multiple membership functions. For the conventional control system, the graphs show the effect of proportional gain on the overshoot and reaching time, as well as the result of the fuzzy control system with multiple member functions. Table 1 shows the detailed values of the statistical analysis. The overshoot increases with the proportional gain of the 
conventional controller to a certain point, approximately $\mathrm{p}=0.9$, while the fuzzy system with multiple membership functions exhibits the smallest overshoot. The reaching time for the conventional control system decreases with increasing proportional gain, while the fuzzy control system with multiple membership functions exhibits well averaged reaching time. Comparing Figure 4 and 5A, we conclude that the damping increases with the proportional gain, and the fuzzy system with multiple membership functions exhibits the modest damping.

For the fuzzy control system, the internal parameters that cause the decrease in overshoot lead to an increase in reaching time, which is in accordance with the intrinsic property of the proportional controller. Although the fuzzification function changes the parameters, the fuzzy control system with multiple membership functions still adopts the proportional controller for the control loop. The decrease in overshoot requires smaller proportional gain - which leads to lower system response, i.e., longer reaching time. By tuning the membership function of fuzzification, i.e., by varying the values of the fuzzification function parameters, the desired balance between system overshoot and reaching time can be achieved.

\section{Conclusion}

Toward an insect leg actuator based on electrical stimulation of leg muscles, a fuzzy control system with multiple membership functions was developed and demonstrated to regulate a beetle's leg to follow a predetermined motion path. The hysteresis was found in the proportional correlation between the frequency of the muscle electrical stimulation and the angular displacement of the stimulated leg. For that, instead of the conventional proportional control system, the fuzzy control system was adopted to regulate the angular displacement and it achieved a smaller overshoot, shorter reaching time, and less damping effect of fluctuation.

\section{Acknowledgements}

This work has been supported by Nanyang Assistant Professorship (NAP), Agency for Science, Technology and Research (A*STAR) Public Sector Research Funding (PSF), and A*STAR-JST (The Japan Science and Technology Agency) joint grant. The authors offer their appreciation to Mr. Chew Hock See, Mr. Cheo Hock Leong, Mr. Ow Yong See Meng, Ms. Chia Hwee Lang, Mr. Roger Tan Kay Chia at School of MAE, NTU. The authors thank Professor Pieter Abbeel (UC Berkeley), Professor Kazuo Ikeda (City of Hope Medical Center), and Professor Michel M. Maharbiz (UC Berkeley) for their helpful advice, and Professor Kris Pister (UC Berkeley) for providing the wireless communication device.

\section{Author contributions}

CZ, FC, TTVD, YL and HS designed research, CZ, FC and HS performed research; CZ, FC and $\mathrm{HS}$ analyzed data; CZ, FC and HS wrote the paper.

\section{Additional Information}

Competing financial interests: The authors declare no competing financial interests. 


\section{Figure Legends}

Figure 1. Experiment materials and devices. (A) Anatomical view of the protraction/retraction pair of muscles controlling the front leg's angular motion. The positions of the implanted stimulation electrodes, namely thin silver wires, are indicated by red crosses. (B) The hardware setup for the closed-loop control system and the 3D motion-detection system. A laptop with a circuit board is used for the closed-loop control system. One desktop with camera sets is used as the 3D motiondetection system. (C) The positions of the markers placed on the beetle, which are recognized by the 3D motion-detection system as point objects. (D) The representation of the recognized markers on the 3D motion detection system. The femur-tibia section of the beetle's leg is represented by the solid line segment joining the two markers placed on the front leg. The lower point indicates the body of the beetle.

Figure 2. Hysteresis motion of electrically stimulated insect-leg. Hysteresis motion for angular displacement corresponding to different stimulation frequencies of two motion types-movement toward and movement away from the neutral position predefined manually ( $N=5, n=25)$. The stimulation frequency starting from $10 \mathrm{~Hz}$ is continuously increased to $120 \mathrm{~Hz}$ and continuously decreased to $10 \mathrm{~Hz}$, with a $10 \mathrm{~Hz}$ step. The stimulation remains at each stimulation frequency for $800 \mathrm{~ms}$. (A) and (B) are reproduction from the authors' previous work in [20] with permission under the terms of the Creative Commons Attribution License in that journal.

Figure 3. Schematic representation of the fuzzy control system. (A) Schematic representation of the fuzzy control system with a single membership function, which is a proportional controller. Instantaneous marker positions are used as the system's feedback information input for adjusting the stimulation frequency as the system's output. (B) Schematic representation of the fuzzy control system with multiple membership functions. Each membership function is a proportional controller. Instantaneous marker positions are used as the system's feedback information input.

Figure 4. Result of conventional control system with single proportional controller. The comparison between the actual leg motion path (red line) and predetermined motion path (blue line). As the Kp value increases, the leg response overshoot generally increases, whereas the settling time decreases $(N=5, n=35)$.

Figure 5. Result of fuzzy control system with multiple membership functions. (A) The comparison between the actual leg motion path (red line) and the predetermined motion path (blue line). The stimulation frequency of two stimulation channels is presented in the same time line as the angular displacement graph $(\mathrm{N}=$ $5, n=35$ ). (B) Fuzzy controller data result example demonstrates how we interpret the presented experimental data. Overshoot and reaching time are defined accordingly.

Figure 6. Comparison of overshoot and reaching time between fuzzy control and conventional control. (A) The graphs provide statistical analysis of the overshoot results in both absolute angle displacement and percentage $(N=5, n=$ 
35). The blue bar graph indicates the average value, and the error indicates the deviation. The first blue bar graph shows the result of the fuzzy system with multiple membership functions. The blue bar graphs after the first one show the result of the fuzzy system with a single membership function at each $\mathrm{Kp}$ value from repeated experiments. (B) The graph provides statistical analysis of the reaching time results in millisecond $(\mathrm{N}=5, \mathrm{n}=35)$. The blue bar graph indicates the average value, and the error indicates the deviation. The first blue bar graph shows the result of the fuzzy system with multiple membership functions. The blue bar graphs after the first one show the result of the fuzzy system with a single membership function at each $\mathrm{Kp}$ value from repeated experiments. 


\section{References}

1. Gross, M., Towards living machines. Current biology : CB, 2013. 23(18): p. R821-R823.

2. Akiyama, Y., et al., Room temperature operable autonomously moving biomicrorobot powered by insect dorsal vessel tissue. PLoS One, 2012. 7(7): p. e38274.

3. Altendorfer, R., et al., RHex: A Biologically Inspired Hexapod Runner. Autonomous Robots, 2001. 11(3): p. 207-213.

4. Bozkurt, A., J. F. Gilmour R, and A. Lal, Balloon-assisted flight of radio-controlled insect biobots. IEEE Trans Biomed Eng, 2009. 56(9): p. 2304-7.

5. Daly, D.C., et al., A Pulsed UWB Receiver SoC for Insect Motion Control. IEEE Journal of Solid-State Circuits, 2010. 45(1): p. 153-166.

6. Hinterwirth, A.J., et al., Wireless stimulation of antennal muscles in freely flying hawkmoths leads to flight path changes. PLoS One, 2012. 7(12).

7. Holzer, R. and I. Shimoyama. Locomotion control of a bio-robotic system via electric stimulation. in Intelligent Robots and Systems, 1997. IROS '97., Proceedings of the 1997 IEEE/RSJ International Conference on. 1997.

8. Sato, H., et al., Remote radio control of insect flight. Front Integr Neurosci, 2010. 3.

9. Tsang, W.M., et al., Flexible split-ring electrode for insect flight biasing using multisite neural stimulation. IEEE Trans Biomed Eng, 2010. 57(7): p. 1757-64.

10. Tubbs, T.B., A.N. Palazotto, and M.A. Willis, Biological Investigation of Wing Motion of the Manduca Sexta. International Journal of Micro Air Vehicles, 2011. 3(2): p. 101118.

11. Wang, H., N. Ando, and R. Kanzaki, Active control of free flight manoeuvres in a hawkmoth, Agrius convolvuli. J Exp Biol, 2008. 211(Pt 3): p. 423-32.

12. Wessnitzer, J. and B. Webb, Multimodal sensory integration in insects--towards insect brain control architectures. Bioinspir Biomim, 2006. 1(3): p. 63-75.

13. Cheng, B., X. Deng, and T.L. Hedrick, The mechanics and control of pitching manoeuvres in a freely flying hawkmoth (Manduca sexta). J Exp Biol, 2011. 214(Pt 24): p. 4092-106.

14. Maharbiz, M.M. and H. Sato, Cyborg Beetles. Scientific American, 2010. 303(6): p. 94-99.

15. Sato, H., et al., A cyborg beetle: Insect flight control through an implantable, tetherless microsystem. Mems 2008: 21st leee International Conference on Micro Electro Mechanical Systems, Technical Digest, 2008: p. 164-167.

16. Sato, H., et al., Deciphering the Role of a Coleopteran Steering Muscle via Free Flight Stimulation. Current Biology, 2015. 25(6): p. 798-803.

17. Sato, H., et al., Cyborg Beetles: The Remote Radio Control of Insect Flight. 2010 leee Sensors, 2010: p. 1-4.

18. Sato, H., et al., Radio-Controlled Cyborg Beetles: A Radio-Frequency System for Insect Neural Flight Control. leee 22nd International Conference on Micro Electro Mechanical Systems (Mems 2009), 2009: p. 216-219.

19. Thang, V.D.T., et al., Insect-Machine Hybrid System. 2013 35th Annual International Conference of the leee Engineering in Medicine and Biology Society (Embc), 2013: p. 2816-2819.

20. Cao, F., et al., A biological micro actuator: graded and closed-loop control of insect leg motion by electrical stimulation of muscles. PLoS ONE, 2014. 9(8): p. e105389.

21. Marzullo, T.C. and G.J. Gage, The SpikerBox: a low cost, open-source bioamplifier for increasing public participation in neuroscience inquiry. PLoS One, 2012. 7(3): p. e30837.

22. Feng, C., et al. Insect-machine hybrid robot: Insect walking control by sequential electrical stimulation of leg muscles. in Robotics and Automation (ICRA), 2015 IEEE International Conference on. 2015. 
23. Brown, A.K. and Y. Lu. Performance test results of an integrated GPS/MEMS inertial navigation package. in Proceedings of ION GNSS. 2004.

24. Chowdhary, G., et al. Low Cost Guidance, Navigation, and Control Solutions for a Miniature Air Vehicle in GPS Denied Environments. in Proceedings of 1st Symposium on Indoor Flight Issues, Mayaguez, Puerto Rico. 2009.

25. Lijie, Z. and C. Ji, MEMS-Based Attitude Measurement System for Miniature Air Vehicle [J]. Journal of Vibration, Measurement \& Diagnosis, 2010. 6: p. 026.

26. SHI, S.-h., et al., Design of miniature electronic compass based on GMR sensor. Journal of Mechanical \& Electrical Engineering, 2012. 6: p. 006.

27. Tanenhau, M., et al. Accurate real time inertial navigation device by application and processing of arrays of MEMS inertial sensors. in Position Location and Navigation Symposium (PLANS), 2010 IEEE/ION. 2010. IEEE.

28. Wei-zheng, L.A.-p.Y. and C. Hong-long, Study on Miniature Navigation System Based on MIMU. Journal of Projectiles, Rockets, Missiles and Guidance, 2005: p. S2.

29. Chen, M., et al., Body area networks: A survey. Mobile networks and applications, 2011. 16(2): p. 171-193.

30. Latré, B., et al., A survey on wireless body area networks. Wireless Networks, 2011. 17(1): p. 1-18.

31. Liu, J., et al., Optical receiver front end for optically powered smart dust. International Journal of Circuit Theory and Applications, 2014.

32. Scott, M.D., B.E. Boser, and K.S. Pister, An ultralow-energy ADC for smart dust. SolidState Circuits, IEEE Journal of, 2003. 38(7): p. 1123-1129.

33. Seo, D., et al., Model validation of untethered, ultrasonic neural dust motes for cortical recording. Journal of neuroscience methods, 2015. 244: p. 114-122.

34. Warneke, B., et al., Smart dust: Communicating with a cubic-millimeter computer. Computer, 2001. 34(1): p. 44-51.

35. Akiyama, Y., et al., Culture of insect cells contracting spontaneously; research moving toward an environmentally robust hybrid robotic system. J Biotechnol, 2008. 133(2): p. 261-6.

36. Akiyama, Y., et al., Long-term and room temperature operable bioactuator powered by insect dorsal vessel tissue. Lab Chip, 2009. 9(1): p. 140-4.

37. Sato, H. and M.M. Maharbiz, Recent developments in the remote radio control of insect flight. Front Neurosci, 2010. 4: p. 199.

38. Guschlbauer, C., H. Scharstein, and A. Bueschges, The extensor tibiae muscle of the stick insect: biomechanical properties of an insect walking leg muscle. J Exp Biol, 2007. 210(6): p. 1092-1108.

39. Burns, M.D. and P.N.R. Usherwood, Mechanical properties of locust extensor tibiae muscles. Comparative Biochemistry and Physiology Part A: Physiology, 1978. 61(1): p. 85-95.

40. Debrodt, B. and U. Bassler, Responses of flexor motor neurons to stimulation of the femoral chordotonal organ of the phasmid extatosoma-tiaratum. Zoologische Jahrbucher-Abteilung Fur Allgemeine Zoologie Und Physiologie Der Tiere, 1990. 94(1): p. 101-119.

41. de Haan, A., The influence of stimulation frequency on force-velocity characteristics of in situ rat medial gastrocnemius muscle. Experimental Physiology, 1998. 83(1): p. 77-84.

42. Buschges, A., et al., Synaptic drive contributing to rhythmic activation of motoneurons in the deafferented stick insect walking system. Eur J Neurosci, 2004. 19(7): p. 1856-62. 
43. Malamud, J.G. and R.K. Josephson, Force velocity relationships of a locust flightmuscle at different times during a twitch contraction. Journal of Experimental Biology, 1991. 159: p. 65-87.

44. Stevenson, R.D. and R.K. Josephson, Effects of operating frequency and temperature on mechanical power output from moth flight-muscle. Journal of Experimental Biology, 1990. 149: p. 61-78.

45. Abdulla, S.C., O. Sayidmarie, and M.O. Tokhi, Functional electrical stimulation-based cycling assisted by flywheel and electrical clutch mechanism: A feasibility simulation study. Robot. Auton. Syst., 2014. 62(2): p. 188-199.

46. Brend, O., C. Freeman, and M. French, Multiple-Model Adaptive Control of Functional Electrical Stimulation. Control Systems Technology, IEEE Transactions on, 2015. 23(5): p. 1901-1913.

47. Liao, Y.W., et al., Modeling open-loop stability of a human arm driven by a functional electrical stimulation neuroprosthesis. Conf Proc IEEE Eng Med Biol Soc, 2013. 2013: p. 3598-601.

48. Lynch, C.L., D. Sayenko, and M.R. Popovic, Co-contraction of antagonist muscles during knee extension against gravity: insights for functional electrical stimulation control design. Conf Proc IEEE Eng Med Biol Soc, 2012. 2012: p. 1843-6.

49. Lynch, C.L. and M.R. Popovic, A Comparison of Closed-Loop Control Algorithms for Regulating Electrically Stimulated Knee Movements in Individuals With Spinal Cord Injury. Neural Systems and Rehabilitation Engineering, IEEE Transactions on, 2012. 20(4): p. 539-548.

50. Qin, Z., M. Hayashibe, and C. Azevedo-Coste, Evoked Electromyography-Based Closed-Loop Torque Control in Functional Electrical Stimulation. Biomedical Engineering, IEEE Transactions on, 2013. 60(8): p. 2299-2307.

51. Zhan, L., et al. Real-time closed-loop FES control of muscle activation with evoked EMG feedback. in Neural Engineering (NER), 2015 7th International IEEE/EMBS Conference on. 2015.

52. Popovic, M.R. and T.A. Thrasher, Neuroprosthese. Encyclopedia of Biomaterials and Biomedical Engineering, 2004. 2: p. 1056-1065.

53. Graham, G.M., T.A. Thrasher, and M.R. Popovic, The effect of random modulation of functional electrical stimulation parameters on muscle fatigue. IEEE Trans Neural Syst Rehabil Eng, 2006. 14(1): p. 38-45.

54. Al-Amood, W.S., H.J. Finol, and D.M. Lewis, Chronic stimulation modifies the isotonic shortening velocity of denervated rat slow-twitch muscle. Proc R Soc Lond B Biol Sci, 1986. 228(1250): p. 43-58.

55. Tanaka, K. and M. Sano. Some properties of fuzzy nonlinear feedback systems. in Industrial Electronics, Control, Instrumentation, and Automation, 1992. Power Electronics and Motion Control., Proceedings of the 1992 International Conference on. 1992. San Diego, CA: IEEE.

56. Tong, R.M. Analysis of closed loop fuzzy systems. in Decision and Control including the Symposium on Adaptive Processes, 1979 18th IEEE Conference on. 1979. Fort Lauderdale, FL, USA: IEEE.

57. Wang, L.-X., Adaptive fuzzy systems and control: design and stability analysis. 1994: Prentice-Hall, Inc. 232.

58. Ying, H., W. Siler, and J.J. Buckley, Fuzzy control theory: A nonlinear case. Automatica, 1990. 26(3): p. 513-520. 
A

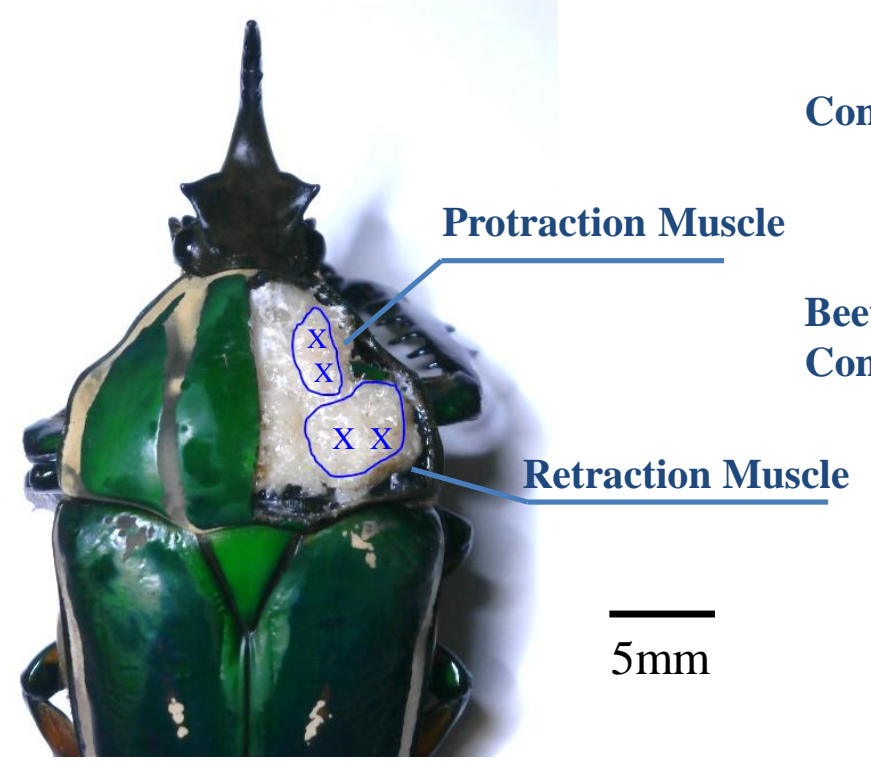

C

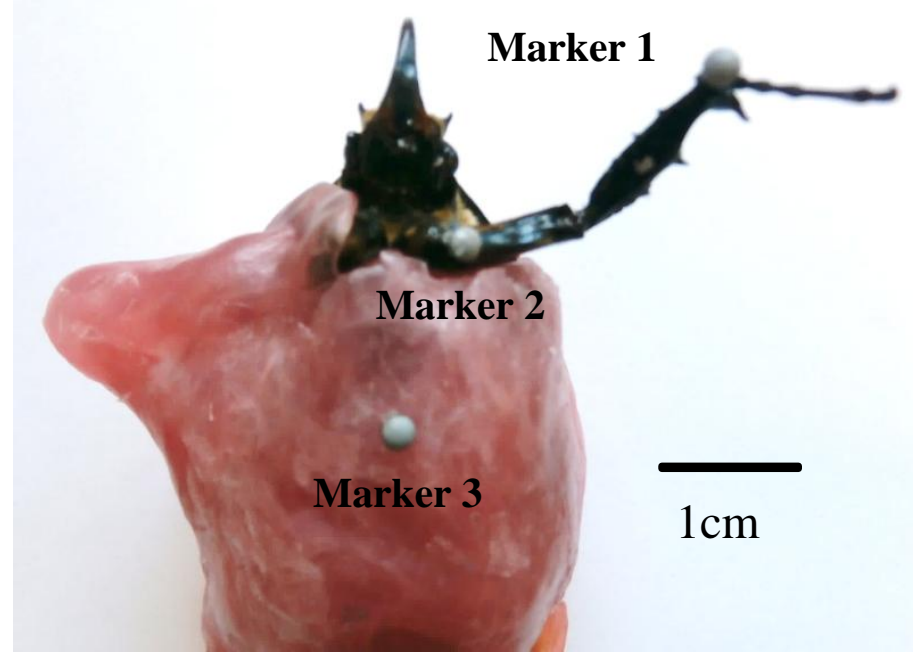

B

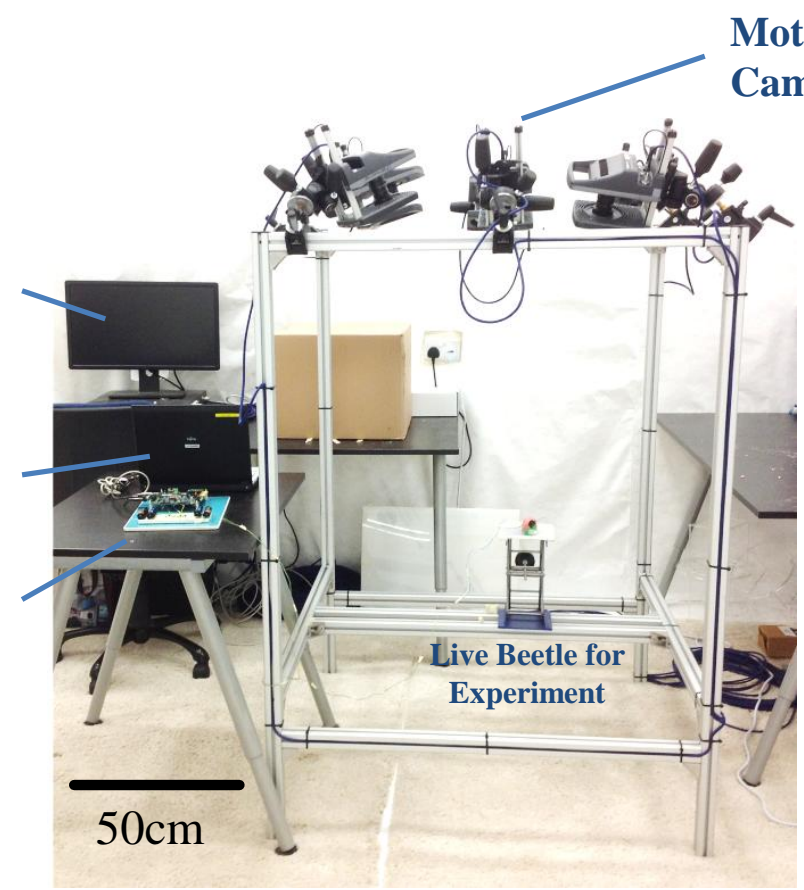

D
Stimulation Circuit

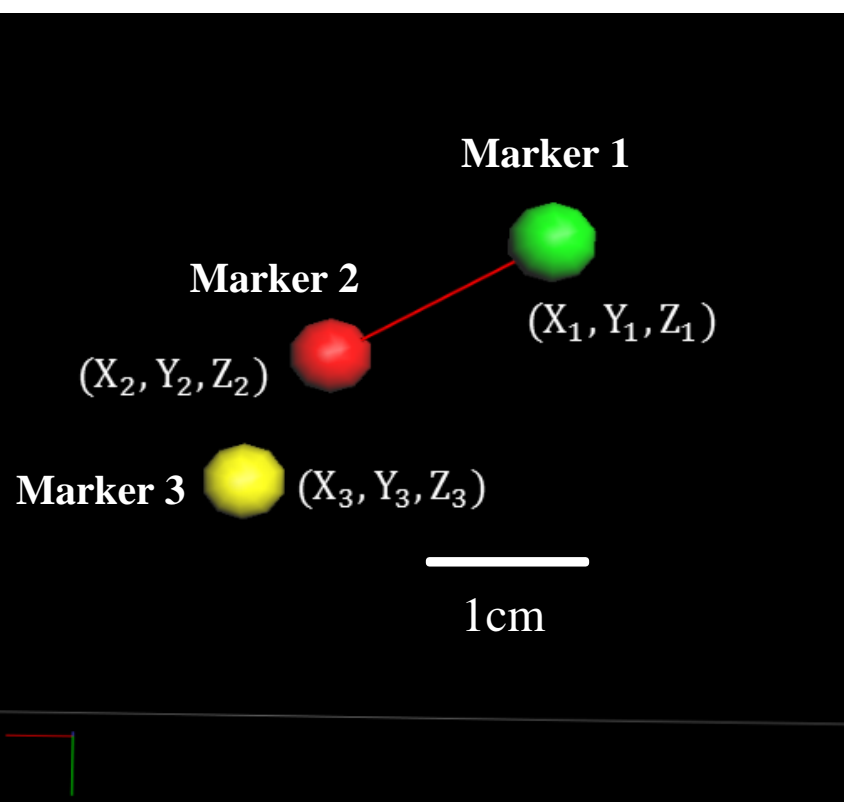

Motion Detection Camera Sets 

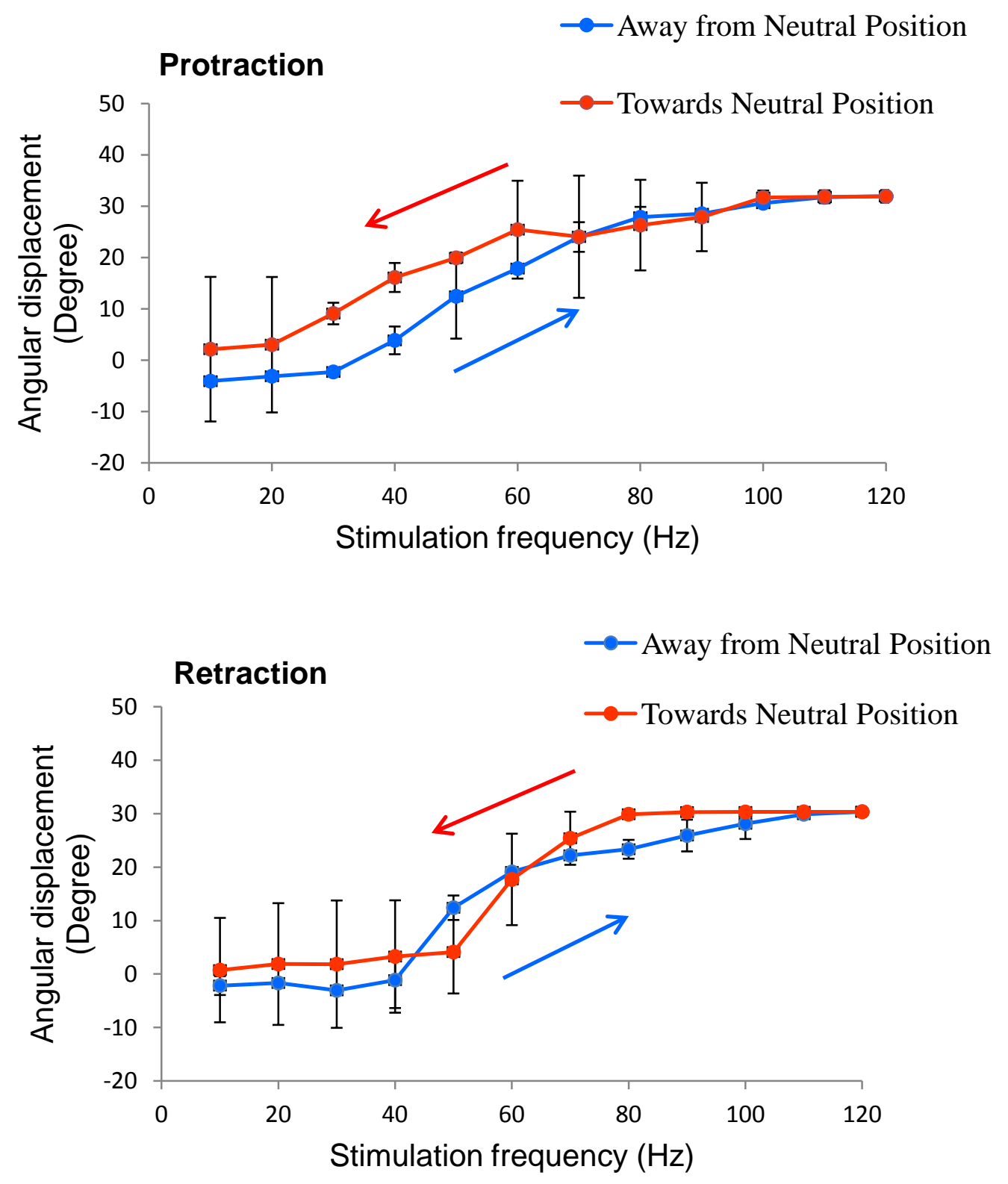
A

Conventional control system with proportional controller

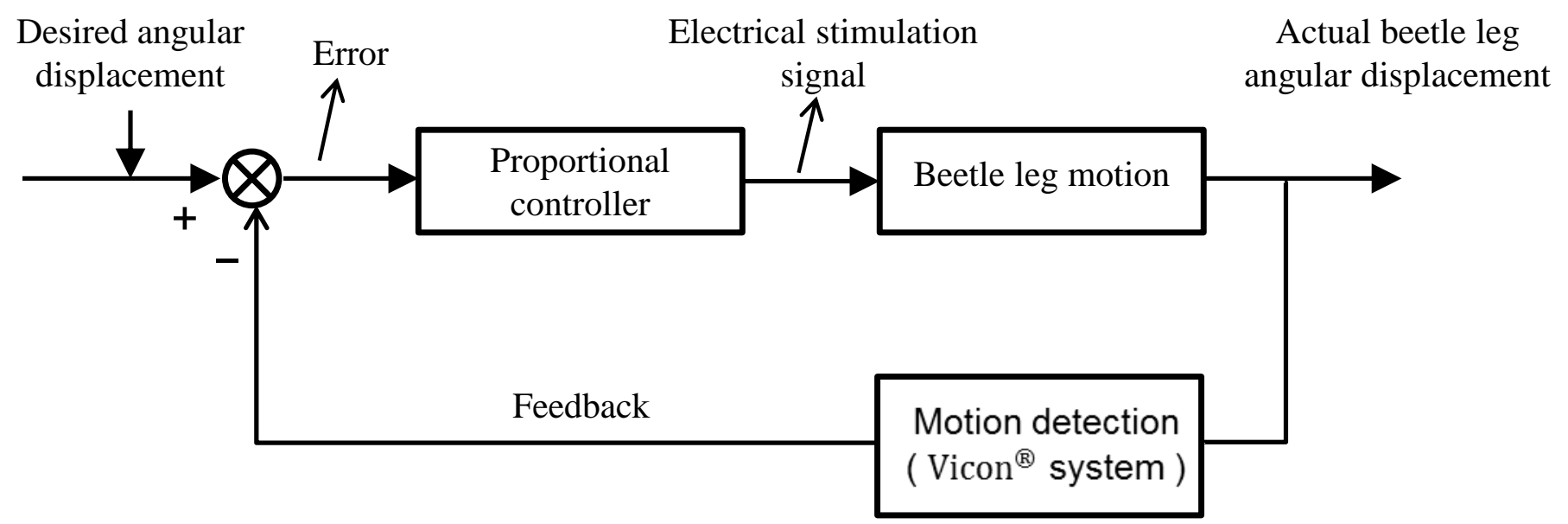

B

Fuzzy control system with multiple membership functions

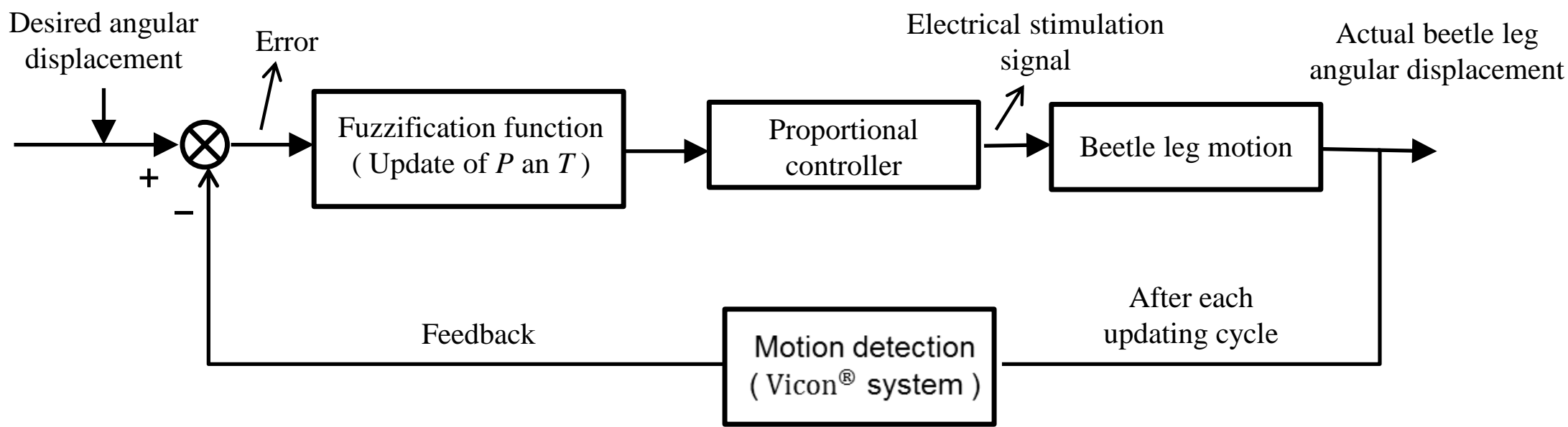

$P / K_{p}=f_{p}\left(\theta_{\text {Desired }}, \theta_{\text {Difference }}\right)$

$T=f_{T}\left(\theta_{\text {Desired }}, \theta_{\text {Difference }}\right)$ 

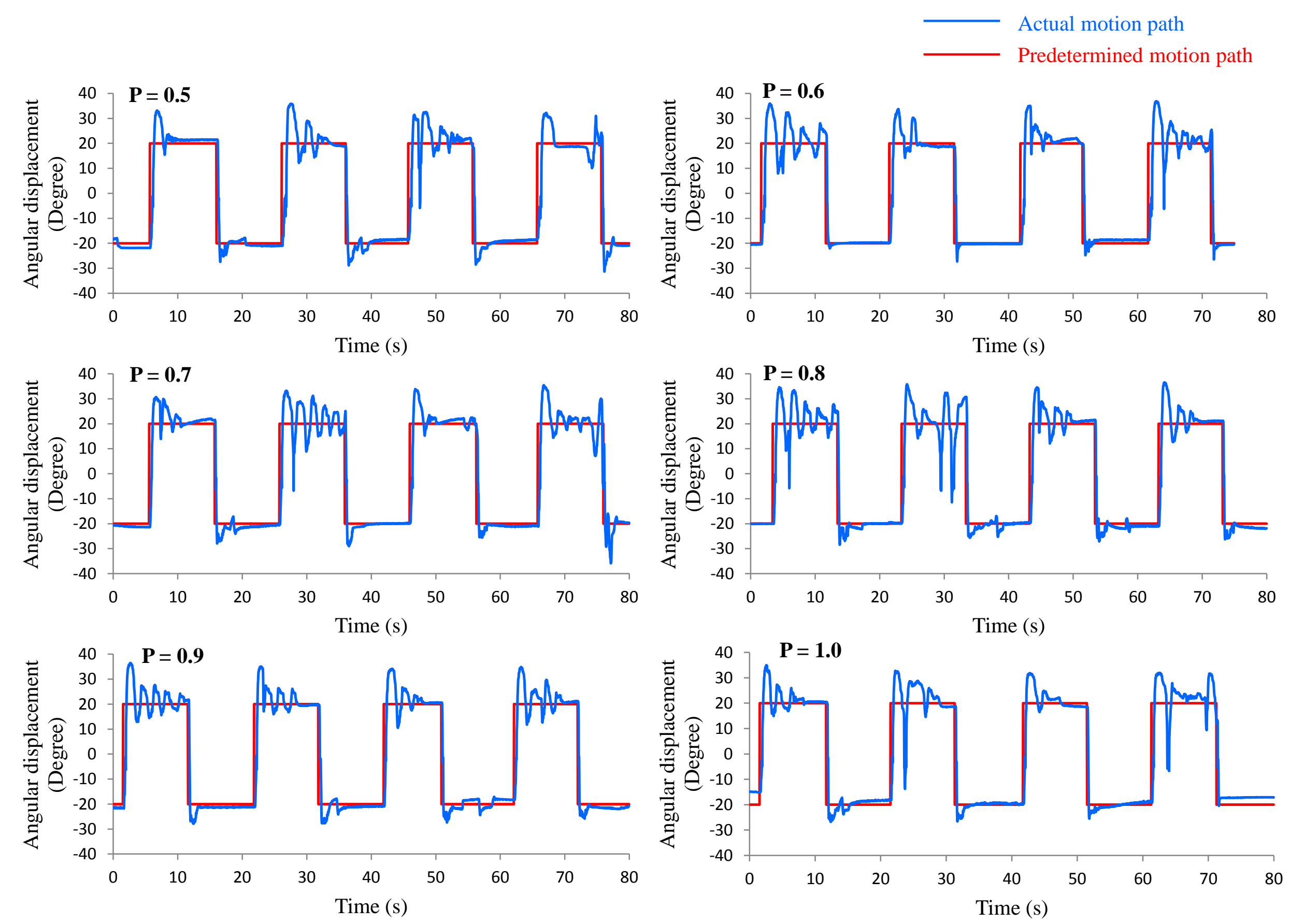
A
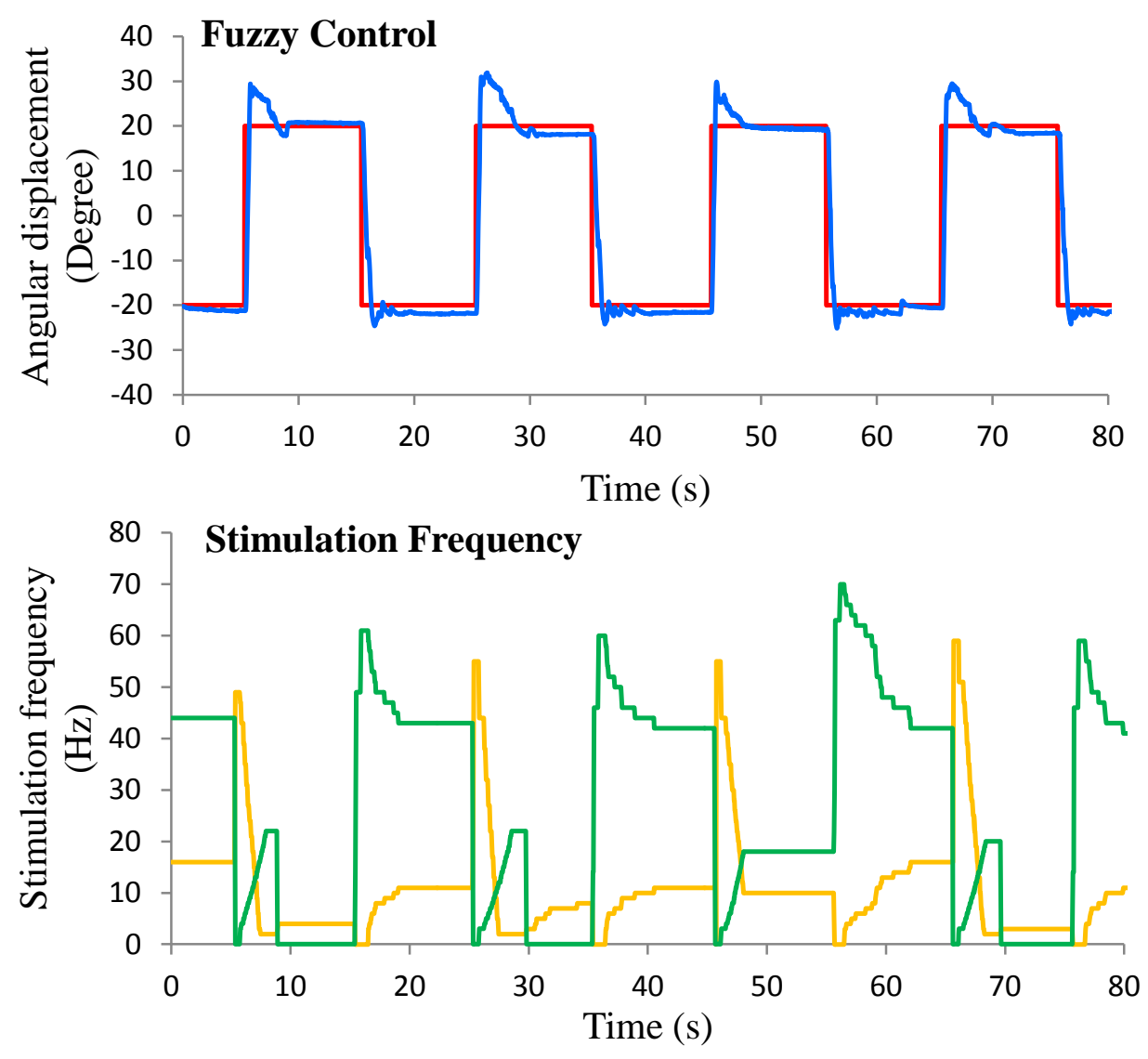
- Stimulation Channel 1 for Protraction

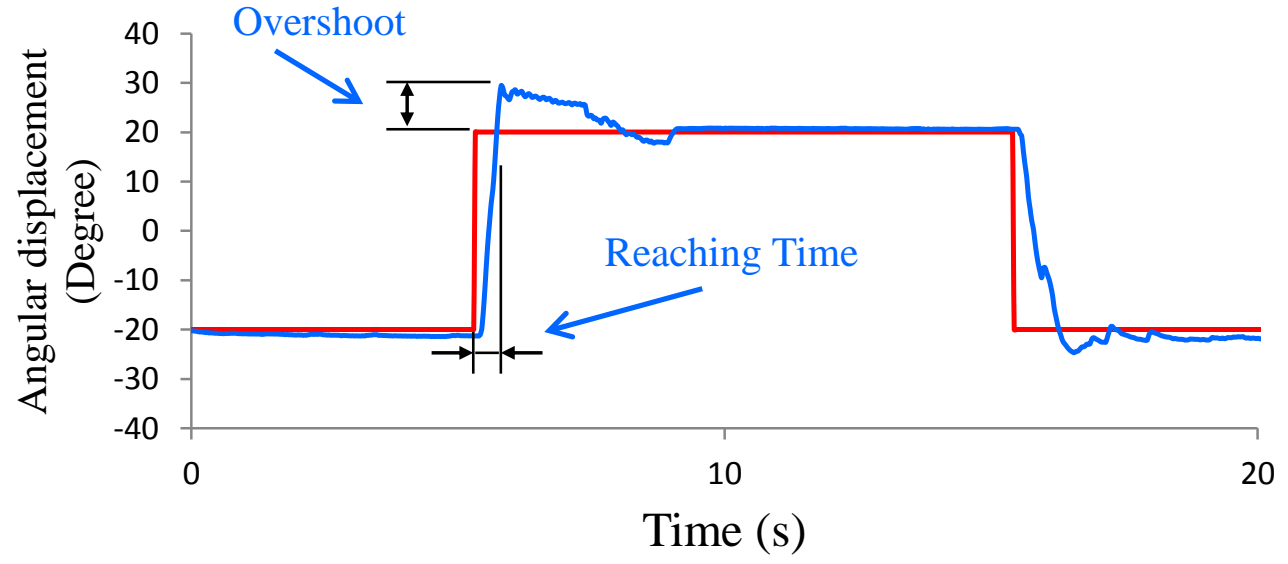




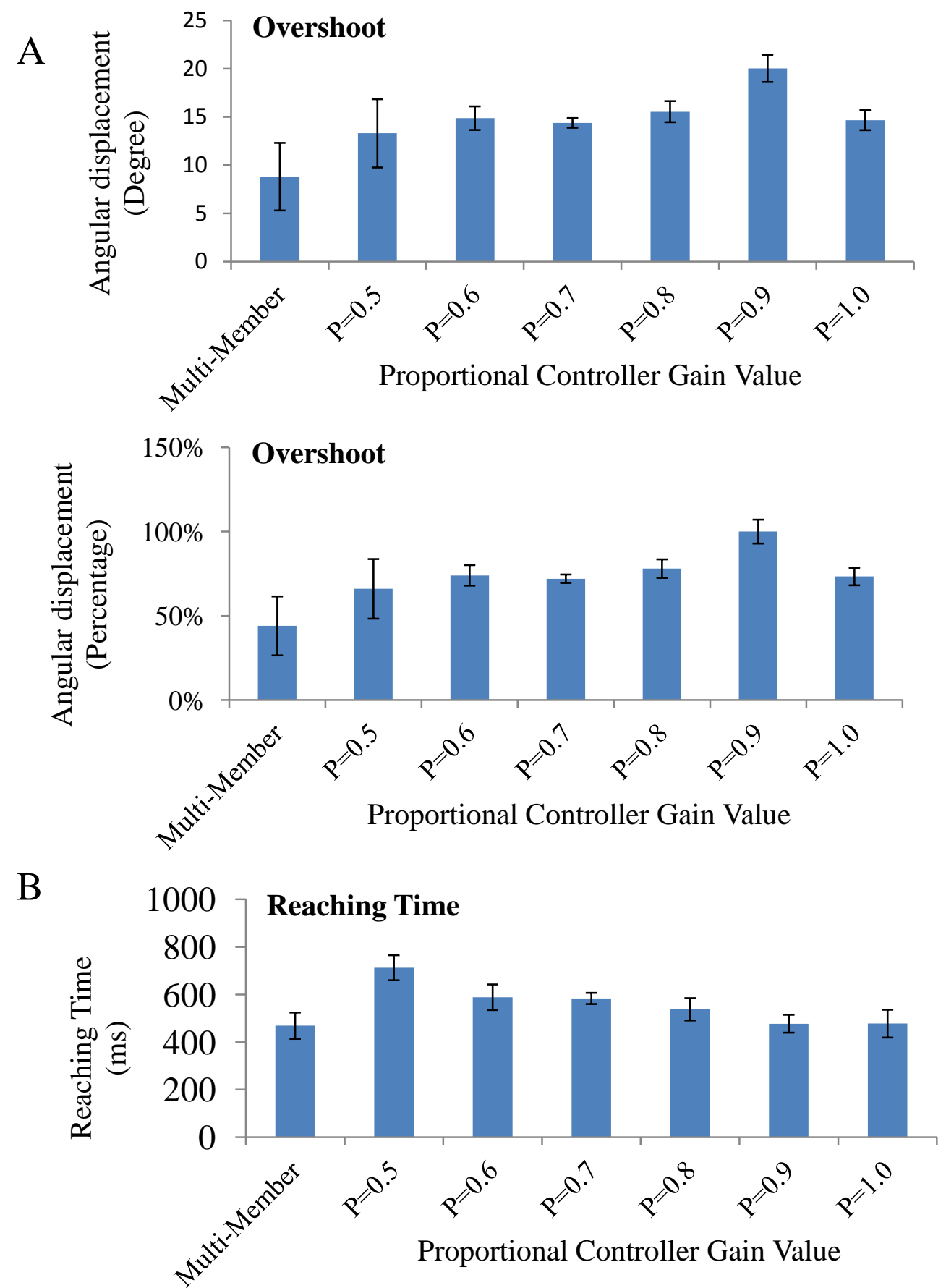




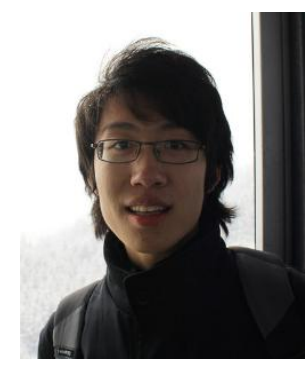

Chao Zhang graduated from Nanyang Technological University with a Bachelor in Mechanical Engineering specialized in Mechatronics, and National University of Singapore with a Master in Statistics and Applied Probability. He worked as a research assistant at Professor Sato's lab on insect-machine hybrid robot system.

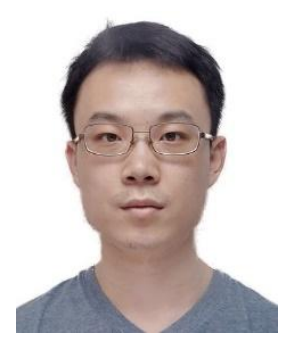

Feng Cao received the B.Eng. degree in Aerospace Engineering from Nanyang Technological University, Singapore, in 2013. He joined Professor Hirotaka Sato's group to pursuit his Ph.D. study at School of Mechanical and Aerospace Engineering, Nanyang Technological University in 2013. His research interest is development of both hardware and software for insect-machine hybrid robot system.

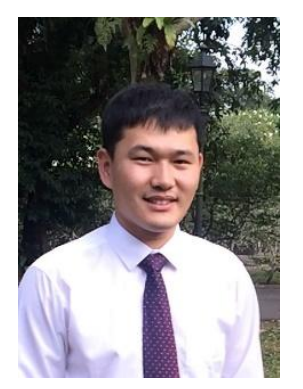

Yao Li graduated from Huazhong University of Science and Technology (China) in Jun, 2013 with a B.Eng. degree in Mechanical Engineering. Then, he joined in Dr. Sato's group at Nanyang Technological University (Singapore) as a Ph.D. student in Aug, 2013. The interests of this group cover a range of topics in insect-machine hybrid system. Currently his research topics are mainly in the insect flight control and insect body attitude analysis.

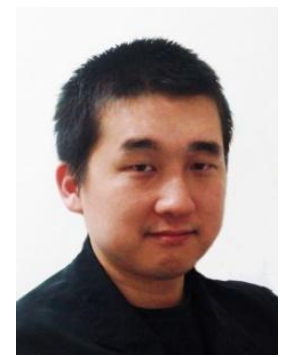

Hirotaka Sato is currently an assistant professor working on insectmachine hybrid robots at School of Mechanical \& Aerospace Engineering, Nanyang Technological University, Singapore. He previously worked on that research at Department of Electrical Engineering and Computer Science at University of California at Berkeley (2008 - 2011) and University of Michigan (2007). He received Ph.D.(2005), Master (2002) and B.S degrees (2000) from Waseda University for his nano/micro fabrication processes for MEMS. 